\title{
Hybrid Transmission Scheme for the Cluster Formation in Wireless Sensor Networks with Noisy Channels
}

\author{
Edgar Romo-Montiel, Mario Eduardo Rivero-Ángeles, Herón Molina-Lozano \\ Instituto Politécnico Nacional, Centro de Investigación en Computación, CDMX, \\ Mexico \\ eromom0900@alumno.ipn.mx, mriveroa@ipn.mx, hmolina@cic.ipn.mx
}

\begin{abstract}
In this paper, we study the performance of an adaptive and fixed transmission schemes where the transmission probability varies according to the evolution of the cluster formation procedure or is fixed for the entire network lifetime, respectively. Also, we propose a hybrid scheme where the transmission probability can either be fixed or adaptive according to the system's conditions. In order to characterize the performance of the proposed scheme, we present a study under non-ideal conditions where noise, interference and fading are present.
\end{abstract}

Keywords: Hybrid transmission probability, adaptive transmission probability, wireless sensors networks, clustering, channel errors.

\section{Introduction}

Wireless Sensors Networks (WSNs) are composed by nodes distributed over certain region of interest with the objective of collecting information, typically, physical data. Such information is transmitted to, at least, one base station or sink node by mean of wireless links $[1,2,4]$.

The clustering techniques have shown its benefits in many applications like data classification, networks, among others [7,?]. LEACH [5] is a commonly used clustering protocol where a certain number of clusters are formed, each with a node called cluster head $(\mathrm{CH})$ who gathers the information from the cluster members $(\mathrm{CMs})$ and sends it directly to the sink node. This technique reduces high range transmissions which demand a higher energy consumption to reach the sink through enabling the $\mathrm{CH}$ nodes as local sinks. Thus the role of nodes acting as either CHs or CMs is rotated throughout the operation of the network in order to avoid a fast energy depletion of nodes acting as CHs by the continuous reception of data.

Cluster-based protocols are composed by two phases: Cluster Formation Phase (CFP) and Steady State Phase (SSP). In the former case, the nodes compete to sharing its own information in order to be discovered in the network and create the clusters. In the last one, the clusters have formed and each node 
has assigned to a specific slot to send its collected information periodically to its respective $\mathrm{CH}$ in a contending-free scheme based on TDMA. Indeed, since the nodes transmit data continuously, resources are not wasted as each time slot is used by a particular sensor node inside the cluster. Conversely, at the cluster formation procedure, a random access protocol based on the NP/CSMA protocol is used $[6,8]$.

Since transmissions by each node in CFP only occur at certain moments of whole the process, it is not practical to assign fixed resource to specific nodes. Therefore, it is essential to carefully select the parameters of the random access protocol in order to maintain an acceptable operation of the network in terms of energy consumption and reporting latency.

In this work, we study the impact of the transmission probability of nodes focusing on three transmission probability strategies: fixed, adaptive and hybrid that can be used in a noisy channel in order to improve the performance of the system. In this sense the analysis is based on the reduction of the energy consumed and the cluster formation time, comparing each one under the same parameters. Since the analysis presented here focuses on studying the transmission probability in the random access transmissions in noisy channels, the results can be easily extended to any clustering protocol such as HEED [10].

The rest of the paper is organized as follows. In section 2 we present the system model, the clustering scheme, the adaptive transmission scheme, the hybrid scheme and the general assumptions. Then, in Section 3, we describe the parameters used in order to analyze the proposed protocol and in Section 4 we present numerical results that characterize the overall performance of the WSN. Finally, Section 5 presents the conclusions.

\section{System Model}

The cluster formation phase is the crucial state to the correct functioning of the network because it is the first step to organize the network. Base on that, it is important to choose correct parameters to establishing the network.

Independently of what algorithm to build the clusters is chosen, each node have to transmit a Hello Package (HP) with its own data in order to be discovered in the network. The main issue is that those transmissions are done inside a contending scheme, therefore a set of collisions and wasted time slots are susceptible to occur because there is no assigned specific resources for nodes. Specifically, all nodes that have not successfully transmitted their HP transmit in each time slot with probability $\tau$.

Additionally, the nodes have to deal with the errors that are present in wireless channels like noise, fading or interferences. These errors are the cause of miss detections and overhearing errors [3]. Building from this, the effect of a noisy channel in the packet transmission is modeled as two types of error probabilities: False Positive Probability $(\mathrm{Pe}+)$ which means that the sink detected a successful transmission that in fact did not happened, and False Negative Probability (Pe-) 
when a successful transmission is not detected by the sink due to fading channels or to obstacles or noise in the transmission trajectory.

Then, the system performance is studied for a wide range of these error probabilities. As its name describes, in Fixed Transmission Scheme (FTS), the value of $\tau$ is the same for all the nodes along the network lifetime. Under this assumption, each node will transmit all the time with probability $\tau=1 / N$, where $N$ is the initial number of nodes in the network.

In our Adaptive Transmission Scheme (ATS), the value of $\tau$ is not fixed, but rather it varies according to the number of nodes that have already transmitted their HP successfully. As such, at the beginning of the CFP, nodes transmit with a low value of $\tau$ in order to reduce collisions, and it is increased at the end of this phase, since at this point, there are only a few nodes attempting to do a transmission. Note that a low value of $\tau$ at the end of the cluster formation phase entails a longer number of empty time slots and hence unnecessary energy consumption due to long idle listening times. From this, we noticed that an ATS can set the value of $\tau$ in such a way that it is inversely proportional to the number of nodes attempting to transmit. For each HP successfully received at the sink, the sink sends an ACK packet indicating to all the network that a node has been correctly detected in the cluster formation procedure. Then, all nodes in the system can calculate the current transmission probability $\tau$ value as $\tau_{A}=1 / n^{\prime}$, where $n^{\prime}$ is the estimation of nodes attempting to transmit. And the initial value is $\tau_{A}=1 / N$.

The main disadvantage of this scheme is that, in a noisy channel, the actual number of nodes may be considerably different than the number of nodes estimated in the system. Consider the case where the sink estimates a valid HP when in fact no node transmitted. This case can occur when a interfering neighbor network performed a transmission, that can happen with non-zero probability since the majority of WSNs are established in unlicensed frequency that are commonly saturated by WiFi, bluetooth users or even other sensor networks. In this case, the transmission probability is increased while the number of nodes remained unchanged, causing a higher collision probability scenario. If this happens many times in a short period of time, the performance of the system can be seriously degraded due to the high number of collisions, as we have witnessed in our experiments.

Note the case where the estimated number of remaining nodes is one. Then, the value of $\tau_{A}$ would also be one. In this case, if there are at least two nodes trying to transmit, they will do so with probability one in all subsequent time slots, generating collisions with probability one and hence the clusters would never be formed and these two nodes will run out of energy. A simple solution is to establish a probability transmission threshold $\tau_{t h}$ (in this study it is fixed to 0.45 )

In order to mitigate the previously mentioned effect of the channel errors in the adaptive scheme, we propose a Hybrid Transmission Scheme (HTS) that considers explicitly the presence of errors in the following manner: using ATS when the errors are low i.e. Pe- and Pe+ are pretty small, and FTS when the 
channel presents a burst of errors and both $\mathrm{Pe}-$ and $\mathrm{Pe}+$ are high.

The three schemes are studied under a channel that presents burst of errors as an abstraction of environments that does not have a constant behavior like a factory where power on engines causes a noisy channel, but those engines, or even some machines, are not working all the time.These events produce such burst of errors.

These bursts of errors are considered as a period of time in which the channel presents a probability of error relatively high for both false positive and false negative probabilities (PePmax and PeNmax). The time between burst is exponentially distributed with mean $1 / \lambda$ and each burst has a random duration exponentially distributed with mean $1 / \mu$. Notice that $\lambda$ in Markovian systems is the arrives rate which indicates the mean speed in that the clients arrive to the system, in this context, $\lambda$ is the velocity in which the error busts arrive to the channel. It is important to note that the time intervals between two periods of high error is considered as a low-error probability period in which both false positive and false negative error probabilities are small (PePmin and PeNmin).

\section{System Parameters}

In order to study the performance of these schemes, we performed extensive simulations to observe the network behavior. The system is evaluated through the average energy consumption and the cluster formation delay (cluster formation time) as performance metrics. Also, we considered different network conditions in terms of the number of nodes, probabilities of false positive and false negative errors and value of $\lambda$ and $\mu$ for error bursts.

To evaluate the energy consumption we used the following model: Whenever a node performs a transmission it consumes $E_{t x}$ units of energy, while for any reception each node consumes $E_{r x}$ units of energy. It is important to emphasize that we assume that the nodes have a single transmission and reception power level. Note that the simplicity of this model allows to describe practical scenarios because the respective values of $E_{t x}$ and $E_{r x}$ can be obtained from direct measurements of the energy consumed in commercial devices. In this work is considered the relation of energy consumed by a transceiver MRF24J40MA [9] such that is $E_{t x}=1.2 E_{r x}$. Note that this value is used just as reference. The main parameters of the system are given in Table (I).

Table 1. System Parameters.

\begin{tabular}{|c|c|}
\hline Parameter & Value \\
\hline Network area & 100 square meters \\
\hline $\mathrm{N}$ (Nodes in the network) & $5,20,50,100$ \\
\hline Value of PePmax and PeNmax & $0.1,0.2,0.5$ \\
\hline Value of PePmin and PeNmin & 0.01 \\
\hline Energy consumption & $E_{r}=0.01$ units, $E_{t}=0.012$ units \\
\hline Value of $\lambda$ and $\mu$ & $.1, .5,1,1.5,2,3,4,5,10,15$ \\
\hline
\end{tabular}




\section{Numerical Results}

Through the performance of many simulations, we obtained the following results: Fig. 1 shows the system performance with 5 nodes in the network and 0.5 of maximum error probabilities in terms of cluster formation time (a) and the average energy consumption (b). As it shows, the best results, lowest energy consumption and cluster formation times are for the ATS followed by HTS that, in fact, is lightly better than the FTS which had the worse performance. Notice that the system's performance is not affected by the duration of the error bursts.
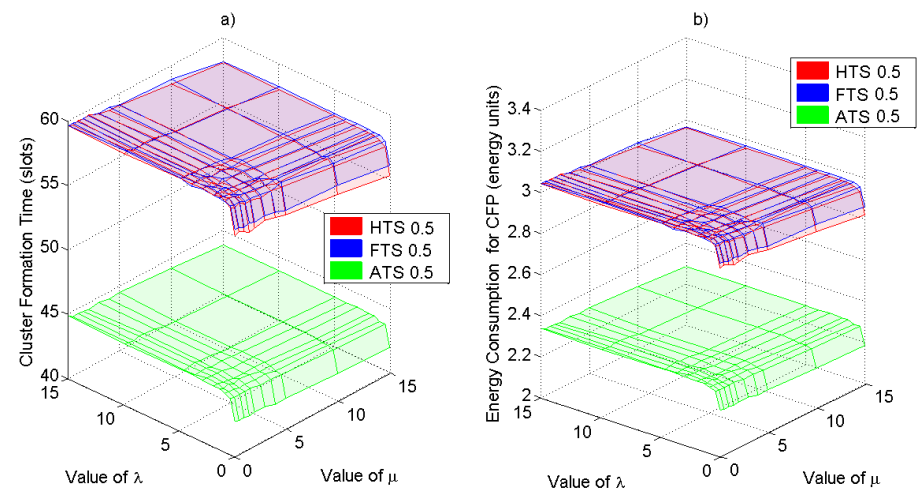

Fig. 1. a) Cluster formation time, b) Energy Consumption for 5 nodes network, $\mathrm{PePmax}=\mathrm{PeNmax}=0.5$.

Fig. 2 and Fig. 3 keep the same behavior but with a higher delay and energy consumption levels. Such results were obtained for a 20 nodes network with maximum error probabilities of 0.1 and 0.2 respectively.

Up to this point, it is clear that ATS has better performance compared to FTS and HTS. Specifically, when the error probabilities in the system are relatively low (although a 0.5 value is considerably high), ATS is working in favorable conditions. However, when the error probability increases to 0.5 the same network changes its behavior regarding to each scheme, such that the best performance is obtained when it works under FTS followed closely by HTS, the last place was for ATS which has a worse performance than the two previous schemes and decrease as the length of the bursts increases. This result is shown in Fig. 4.

For more dense networks, the error presence has a higher impact in the system performance specially to whether it works with ATS and HTS. The results of a network with 50 nodes and maximum error probabilities of 0.1 are shown in Fig. 5 which presents a same form of the graph of the smaller networks and offering better results in ATS upon HTS and FTS. Note that it is more notable the improving of HTS for short error bursts. 

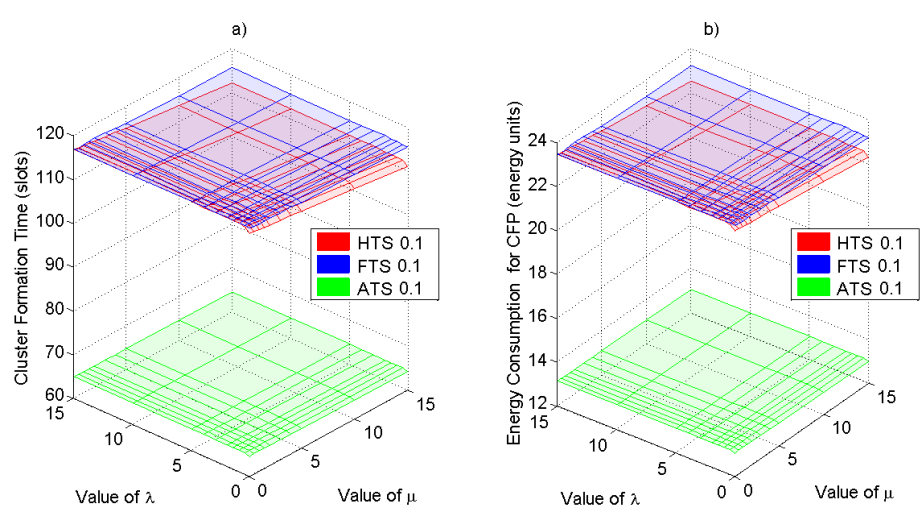

Fig. 2. a) Cluster formation time, b) Energy Consumption for 20 nodes network, $P e P \max =\mathrm{PeNmax}=0.1$.
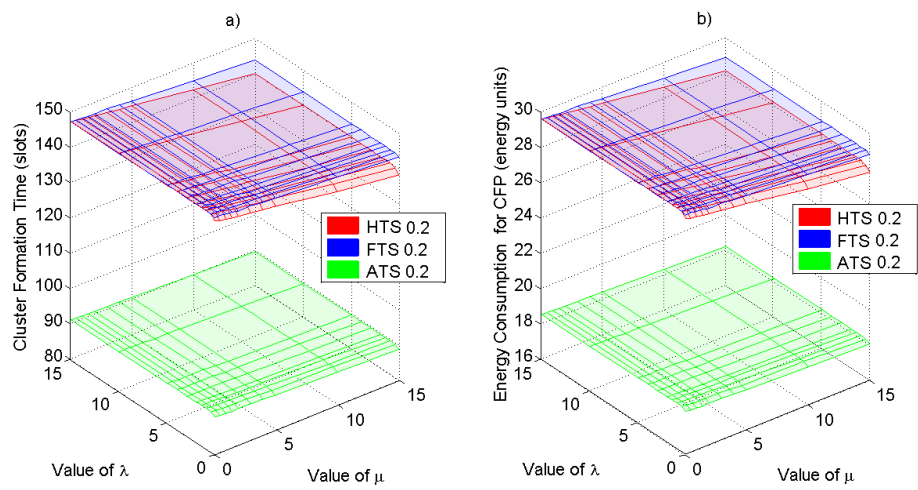

Fig. 3. a) Cluster formation time, b) Energy Consumption for 20 nodes network, $\mathrm{PePmax}=\mathrm{PeNmax}=0.2$.

Fig. 6 and Fig. 7 show the results for the same 50 nodes network and maximum error probabilities of 0.2 and 0.5 respectively. The former shows the best performance for FTS followed by the HTS and ATS. It is important to observe that there is a cross point between ATS and HTS, i. e. for length of the error bursts with value of $\mu \leq 5$ and values of $\lambda \geq 10$. HTS has a better performance upon ATS. The last case still shows the best performance to FTS, followed closely by HTS; ATS has the worse performance which decrease as the length of the error burst increase, namely, both energy consumption and cluster formation time increase as the value of $\mu$ decrease.

The last study was developed for a network with 100 nodes and for maximum error probabilities of $0.1,0.2$ and 0.5 . Fig. 8 shows the results for 0.1 of this network, where we observe that the best performance is for ATS and it is followed by the FTS. However HTS increases the energy consumption and the cluster 

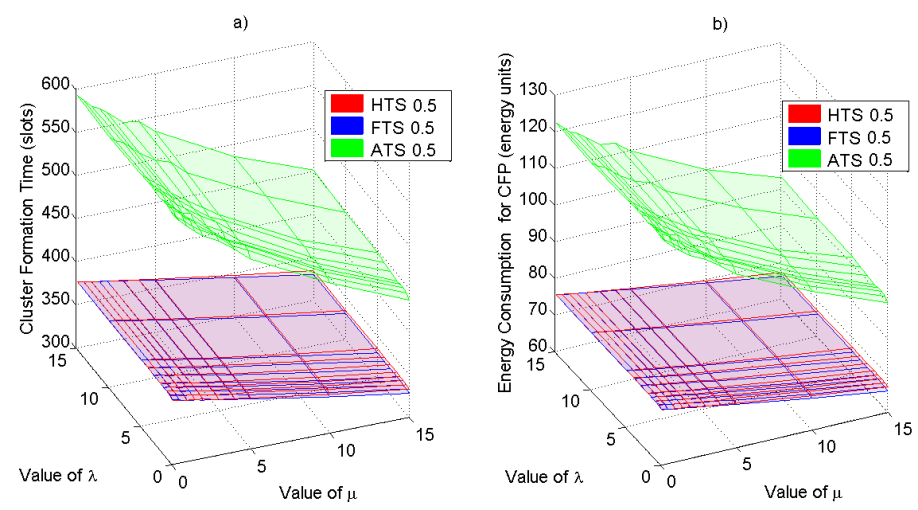

Fig. 4. a) Cluster formation time, b) Energy Consumption for 20 nodes network, $\mathrm{PePmax}=\mathrm{PeNmax}=0.5$.
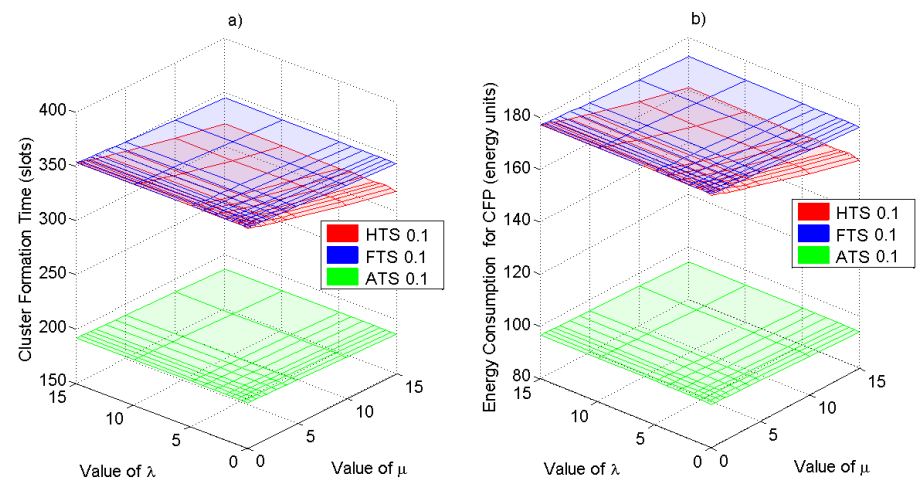

Fig. 5. a) Cluster formation time, b) Energy Consumption for 50 nodes network, $P e P \max =\mathrm{PeNmax}=0.1$.

formation time as the bursts are more frequent and shorter, resulting as the worse scheme.

Finally, in Fig. 9, which corresponds to the 0.2 error probability results, clearly is observed that the best performance is for FTS and the worse is for ATS, independently of the value of $\lambda$ and $\mu$.

Note that this does not contain results for 0.5 since the performance is not acceptable with very high values of both energy consumption and cluster formation delay.

\section{Conclusions}

Through the results we observe that the number of nodes affects directly the system performance independently of errors in the channel and the scheme of 

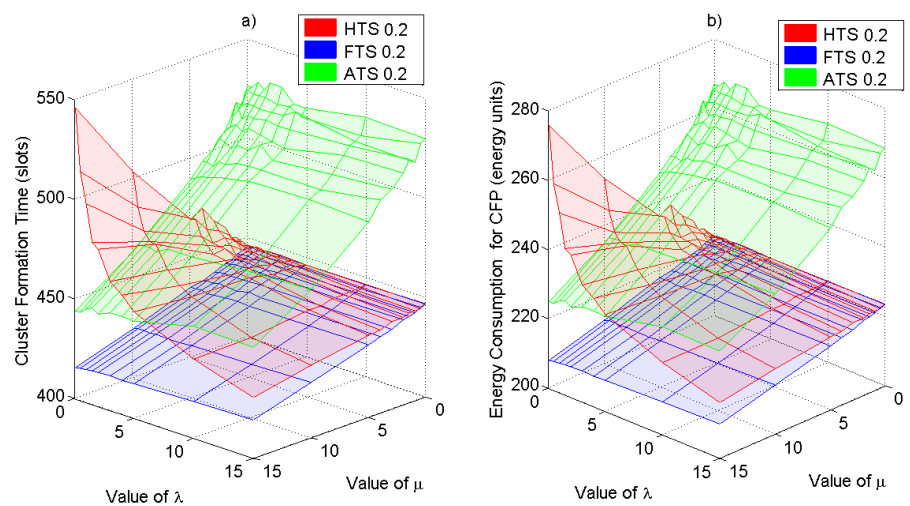

Fig. 6. a) Cluster formation time, b) Energy Consumption for 50 nodes network, $P e P \max =\mathrm{PeNmax}=0.2$.
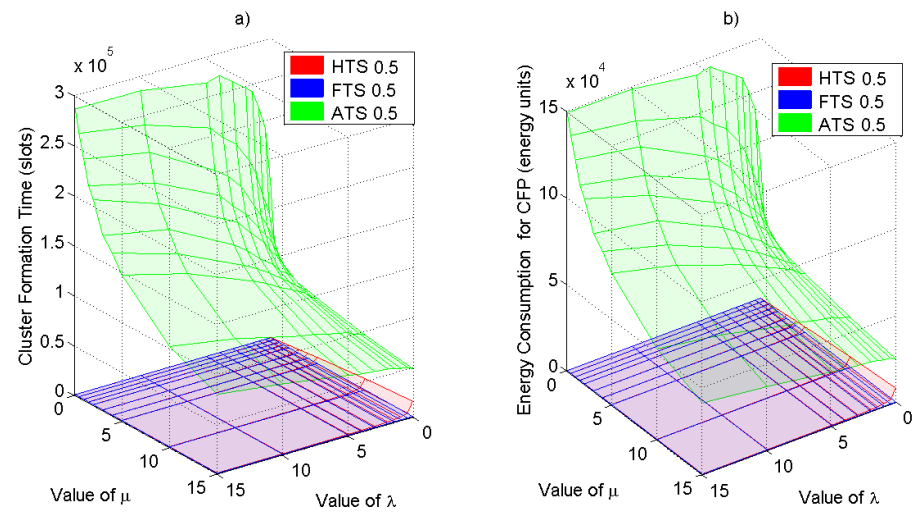

Fig. 7. a) Cluster formation time, b) Energy Consumption for 50 nodes network, $\mathrm{PePmax}=\mathrm{PeNmax}=0.5$.

contending that is adopted, this is quite intuitively because as we have more nodes, we need more transmissions and consequently more energy consumption.

Observing the impact of errors, we note that the three schemes have a different behavior respectively to the error probabilities and the number of nodes. As we see, the adaptive scheme has the best performance as long as the probability PePmax and PeNmax $\leq 0.1$, independently of the number of nodes. On the other hand, ATS is the best proposal for small networks less than 20 nodes and error probability of 0.5 . This is because ATS estimates the current value of $\tau_{A}$ based on the successful transmission, as the channel presents more error, the estimation will no longer be the adequate.

FTS has a better performance in networks larger than 20 nodes and error probabilities higher than 0.1. Comparing to the ATS, FTS is better under hostile channels with error probabilities higher than 0.1 because FTS does not change 

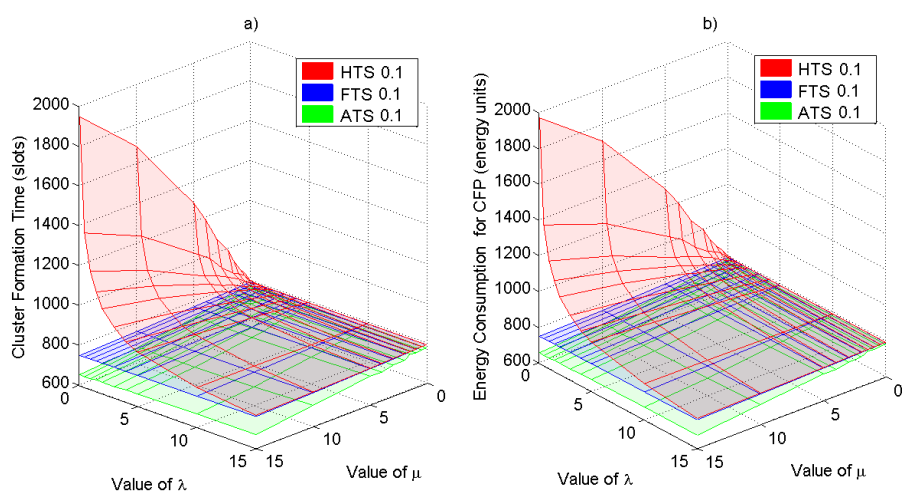

Fig. 8. a) Cluster formation time, b) Energy Consumption for 100 nodes network, $\mathrm{PePmax}=\mathrm{PeNmax}=0.1$.
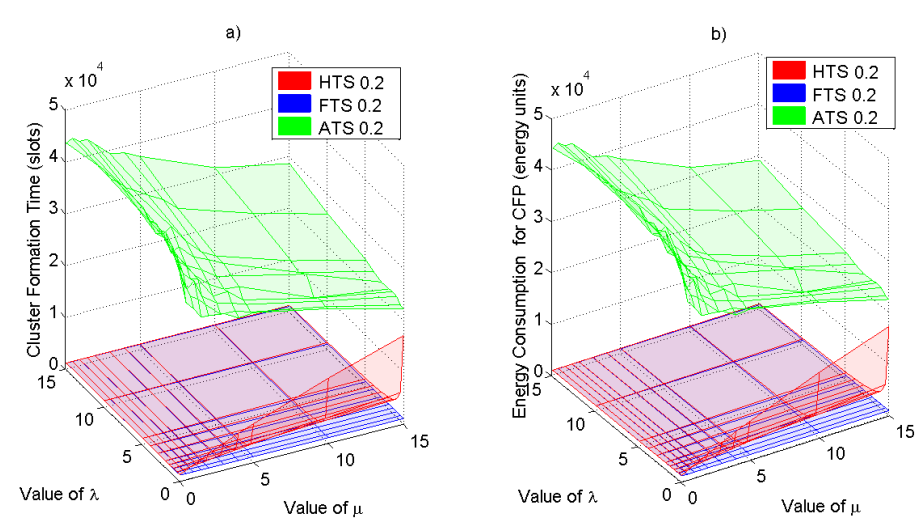

Fig. 9. a) Cluster formation time, b) Energy Consumption for 100 nodes network, $\mathrm{PePmax}=\mathrm{PeNmax}=0.2$.

the value of $\tau$ and it is not affected by the error presences, in this way, it is no feasible that nodes transmit with a higher probability than the optimal which causes collisions and, consequently, wasted time slots.

Finally, HTS, has a behavior similar to FTS. Although in small networks and with less error probabilities HTS is lightly better than FTS, in large networks and bad transmission conditions is closely similar to FTS.

\section{References}

1. Afsar, M.M., Tayarani-N, M.H.: Clustering in sensor networks: A literature survey. Journal of Network and Computer Applications 46, 198 - 226 (2014), http: //www . sciencedirect.com/science/article/pii/S1084804514002124 
2. Ali, A.W., et al.: Energy efficieny in routing protocol and data collection approaches for wsn: A survey. In: International Conference on Computing, Communication \& Automation (ICCCA). pp. 540-545. IEEE (2015)

3. Alnuaimi, M., Shuaib, K., Nuaimi, K., Abdel-Hafez, M.: Performance analysis of clustering protocols in wsn. In: Wireless and Mobile Networking Conference (WMNC), 2013 6th Joint IFIP. pp. 1-6 (April 2013)

4. Azmi, N., Kamarudin, L.M., Mahmuddin, M., Zakaria, A., Shakaff, A.Y.M., Khatun, S., Kamarudin, K., Morshed, M.N.: Interference issues and mitigation method in WSN 2.4GHz ISM band: A survey. In: 2nd International Conference on Electronic Design (ICED). pp. 403-408 (2014)

5. Castalia: Castalia, wireless sensor network simulator (2013), https://castalia. forge.nicta.com.au/index.php/en/

6. Heinzelman, W., Chandrakasan, A., Balakrishnan, H.: Energy-efficient communication protocol for wireless microsensor networks. In: System Sciences, 2000. Proceedings of the 33rd Annual Hawaii International Conference on. pp. 10 pp. vol.2- (Jan 2000)

7. Ke, W., Yangrui, O., Hong, J., Heli, Z., Xi, L.: Energy aware hierarchical clusterbased routing protocol for WSNs. The Journal of China Universities of Posts and Telecommunications 23(4), 46-52 (2016)

8. Kong, L., Xiang, Q., Liu, X., Liu, X.Y., Gao, X., Chen, G., Wu, M.Y.: ICP: Instantaneous clustering protocol for wireless sensor networks. Computer Networks (2016)

9. Koubâa, A., Alves, M., Tovar, E.: Lower protocol layers for wireless sensor networks: a survey. IPP-Hurray Group (2005)

10. Microchip Technology Inc.: MRF24J40MA, Data sheet, $2.4 \mathrm{GHz}$ IEEE Std. 802.15.4 RF Transceiver Module (10 2008)

11. Younis, O., Fahmy, S.: HEED: a hybrid, energy-efficient, distributed clustering approach for ad hoc sensor networks. IEEE Transactions on Mobile Computing $3(4), 366-379$ (2004) 